\title{
Efectividad del tratamiento temprano de niños con mutismo selectivo en los centros educativos
}

\author{
Effectiveness of early treatment of children with selective mutism in \\ educational centers
}

\author{
José Olivares \\ Pablo J. Olivares-Olivares \\ Universidad de Murcia, España \\ Ángel Rosa-Alcázar \\ Universidad Católica de Murcia, España \\ Rec (25 de enero de 2019) Acept (10 de abril de 2019)
}

\begin{abstract}
Resumen
El mutismo selectivo (MS) es un trastorno de ansiedad de baja prevalencia, lo que dificulta su investigación. Pese a su inicio temprano su diagnóstico no suele hacerse antes del acceso a la escolaridad obligatoria. El objetivo de este estudio fue valorar la eficiencia de un protocolo cognitivo-conductual para la intervención psicológica en el contexto educativo (IPMSCE), siguiendo los criterios de la Task Force in Promotion and Dissemination of Psychological Procedures. Participaron 10 niños que presentaban una demora inferior a dos años entre el inicio del MS y su diagnóstico $(1.00 \pm 0.54)$. La edad media fue de 4.94 años y el $80 \%$ fueron niñas. Se utilizó un diseño de acumulación de casos, con medidas del tratamiento antes de su aplicación, al finalizar y en un seguimiento a los 12 meses, obtenidas a través de los padres, los profesores y un test situacional. Los resultados muestran la efectividad de la intervención.

Palabras clave: mutismo selectivo, centros educativos, protocolo de tratamiento cognitivo-conductual.
\end{abstract}

\begin{abstract}
Selective mutism (SM) is an anxiety disorder of low prevalence, which makes its investigation difficult. Despite its early start, its diagnosis is not usually made before access to compulsory schooling. The objective of this study was to assess the efficiency of a cognitive-behavioral protocol for psychological intervention in the educational context (IPMSCE), following the criteria of the Task Force in Promotion and Dissemination of Psychological Procedures. Ten children with a delay of less than two years between the onset of SM and its diagnosis $(1.00 \pm 0.54)$ participated. The average age was 4.94 years and $80 \%$ were girls. A case accumulation design was used, with measures of treatment before its application, at the end and in a followup at 12 months, obtained through parents, teachers and a situational test. The results show the effectiveness of the intervention.

Keywords: selective mutism, educational centers, cognitive-behavioral treatment protocol.
\end{abstract}

Correspondencia: Murcia University, Department of Personality, Evaluation and Psychological Treatment, Campus de Espinardo, 30100 Murcia (Spain). Tel.: +34 868883976; fax: +34 86888 4111, Email addresses: jorelx1@um.es 


\section{Introducción}

El mutismo selectivo (MS) es un trastorno por ansiedad que se inicia generalmente antes de los 5 años (American Psychiatric Asociación [APA], 2013).

$\mathrm{Su}$ inicio ocurre generalmente cuando los niños asisten a los jardines de infancia y parvularios, pero no se suele diagnosticar hasta que no acceden a la escolaridad obligatoria donde la ausencia de habla es un problema para la evaluación de algunas materias escolares (Oerbeck, Stein, Pripp \& Kristensen, 2015).

El MS se caracteriza por un fracaso reiterado en el uso adecuado del habla de los niños/adolescentes cuando tienen que hacerlo en situaciones sociales específicas, pese a que puede verificarse que hablan en otros lugares (APA, 2013).

Su diagnóstico requiere descartar que la ausencia de habla no es parte de su proceso de adaptación (OlivaresOlivares \& Olivares, 2018). Por ello, solo se considerará desadaptativa cuando el mutismo se prolongue durante más de un mes, sin que éste sea el primero tras incorporarse a un nuevo centro educativo o al trasladarse de un centro a otro. Además, el mutismo no debe poder explicarse mejor por efecto de otras variables como un dominio limitado del idioma en el que ha de expresarse (APA, 2013).

Los niños/adolescentes que cumplen los criterios para el diagnóstico de MS pueden no hablar con todos o parte de sus compañeros de clase, con sus cuidadores y/o con sus profesores, mientras que si lo hacen en casa y con sus amigos (Viana, Beidel \& Rabian, 2009).

El hecho de que éstos no se comuniquen verbalmente de manera regular en determinadas situaciones puede tener efectos muy perjudiciales para ellos e incluso resultar crítico si dificulta su desarrollo social y/o limita significativamente sus posibilidades de aprendizaje (Busse \& Downey, 2011).

Bulotsky-Shearer, Fantuzzo \& McDermott (2008) subrayan que los datos muestran que los niños/adolescentes con más y mejores habilidades sociales y escolares son los que con mayor frecuencia están involucrados en las interacciones sociales de alta calidad con sus maestros y compañeros. En cambio, las dificultades para comunicarse verbalmente y desenvolverse correctamente, en entornos de aprendizaje estructurado, predice peores resultados académicos y mayores retrasos en el desarrollo social.

Asimismo, la ausencia del habla durante los primeros años de un niño en los centros educativos (opcionales u obligatorios) resulta especialmente preocupante, porque interfiere de manera relevante en el desarrollo del aprendizaje temprano y en la calidad, variedad y cantidad de las experiencias sociales que se hallan en la base de la construcción de la competencia social.

Dado que los datos muestran que las mejorías y recuperaciones "espontáneas" son raras en el MS (Oerbeck, Overgaard, Stein, Pripp \& Kristensen, 2018), todo lo expuesto pone de manifiesto la necesidad de intervenir lo antes posible para reducir el sufrimiento de los niños y los adultos que interactúan con ellos, así como el deterioro funcional asociado (Olivares \& Olivares-Olivares, 2018).

Pese a que la investigación del tratamiento del MS aplicando protocolos de tratamiento en muestras por acumulación de casos ya cuenta con los primeros resultados en lengua inglesa (Bergman et al., 2013; Oerbeck, Johansen, Lundahl \& Kristensen, 2012), en ensayos clínicos controlados, todavía no disponemos de estudios publicados en español en los que se muestre la bondad de la aplicación de un protocolo de esta naturaleza donde se presenta el MS con mayor frecuencia: el centro educativo.

Nuestro objetivo, en la presente investigación, fue poner a prueba los efectos de la aplicación de un protocolo para el desarrollo de la intervención psicológica en niños con MS en los centros educativos (IPMSCE; Olivares y Olivares-Olivares, 2008b). El contraste de su efectividad se realizó siguiendo los requisitos de la Task Force in Promotion and Dissemination of Psychological Procedures (1995), es decir, cumpliendo el requisito del criterio II que conlleva realizar una serie de experimentos, con diseño de caso único (más de 9), en los que se hayan utilizado estrategias potentes de control experimental.

\section{Método}

\section{Participantes}

Los participantes provienen de una serie consecutiva de niños que se sometieron a la evaluación diagnóstica realizada por psicólogos clínicos, expertos en trastornos de ansiedad en la infancia-adolescencia y miembros 
de la Unidad de Terapia de Conducta (UTECo) de la facultad de psicología de la Universidad de Murcia. Fueron remitidos desde la policlínica El Pla (http://www.clinicaelpla.com/) que tiene un acuerdo de colaboración con la Universidad de Murcia, psicólogos de los servicios de orientación educativa de la Región de Murcia y a través de la web de nuestro grupo de investigación (https:/www.um.es/grupos/grupo-fobiasocial/cientifico.php). Los criterios de inclusión y exclusión fueron:

- Inclusión: cumplir los criterios del DSM-5 (APA, 2013) para un diagnóstico primario de MS, no presentar una demora superior a los dos años desde el inicio del trastorno informado por los padres hasta el momento de solicitar su diagnóstico en el marco de nuestro programa de detección e intervención temprana y hablar al menos con una persona del centro educativo (aunque fuese de manera ocasional, estando solos y al oído).

- Exclusión: presentar una demora superior a los dos años entre el inicio informado por los padres del MS y la solicitud de su diagnóstico por nuestro equipo, que los padres no hablaran fluidamente español, presentar un $\mathrm{CI} \leq 75$, cumplir los criterios para el diagnóstico de un trastorno psicótico o un trastorno generalizado del desarrollo, estar tomando medicación psicotrópica o recibiendo otro tratamiento para el MS.

Se seleccionaron 10 aspirantes (rango: 3.90-6.20 años; Media =4.94, Desviación típica —DT— $=0.89 ; 80 \%$ niñas) que en la Clinician's severity rating (CSR — ADIS-IV-P; Silverman, Albano \& Sandín, 2003-) alcanzaron una severidad media en el MS de $6.30(D T=0.48)$. Véanse en la Tabla 1 sus características.

Tabla 1. Características descriptivas

\begin{tabular}{|c|c|c|}
\hline & & $N=10$ \\
\hline & Edad (Media $\pm D T)$ & $4.94 \pm 0.88$ \\
\hline \multirow{2}{*}{$\underset{0}{\stackrel{0}{0}} \stackrel{0}{e}$} & Hombres & $2(20)$ \\
\hline & Mujeres & $8(80)$ \\
\hline \multicolumn{2}{|c|}{ Demora detección del trastorno $($ Media $\pm D T)$} & $1.00 \pm 0.54$ \\
\hline \multirow{2}{*}{ 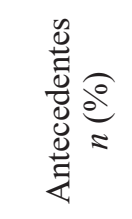 } & Con antecedentes & $1(10)$ \\
\hline & Sin antecedentes & $9(90)$ \\
\hline \multirow{13}{*}{ 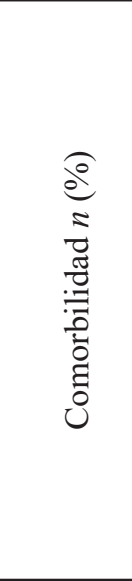 } & Fobias social & $0(0)$ \\
\hline & Fobia específica & $1(10)$ \\
\hline & Ansiedad separación & $5(50)$ \\
\hline & Ansiedad generalizada & $1(10)$ \\
\hline & Ataques pánico & $0(0)$ \\
\hline & Tics & $1(10)$ \\
\hline & Trastorno por déficit atencional & $0(0)$ \\
\hline & Enuresis & $1(10)$ \\
\hline & Encopresis & $1(10)$ \\
\hline & Trastorno negativista desafiante & $0(0)$ \\
\hline & Trastorno obsesivo-compulsivo & $1(10)$ \\
\hline & Depresión & $1(10)$ \\
\hline & Trastorno del habla y el lenguaje & $0(0)$ \\
\hline
\end{tabular}




\section{Evaluación}

Se aplicaron las siguientes estrategias e instrumentos de evaluación:

PRUEba PARA EVALUAR El HABLA EN PRESENCIA DE/CON UN EXTRAÑo en niños con mutismo selectivo (EHEx). Se trata de un test situacional, de 30 minutos de duración, diseñado ad hoc (Olivares \& Olivares-Olivares, 2008c). Su estructura la conforman tres fases (estar padres y niño solos en la sala de espera antes de entrar la persona extraña - A- con el extraño - Ex-, solos en la sala los padres y el niño después de abandonarla el extraño - D-), de 10 minutos de duración cada una. En éstas se observa y registra el modo (gestual, escrito y oral) y el tipo de comunicación (gestos, notas escritas, habla inaudible - cuchicheo-y audible para los observadores, habla como respuesta a una instigación o pregunta y habla espontánea). Se desarrolla en una sala de espera, dotada de espejo bidireccional o de un sistema de grabación audiovisual. Se inicia una vez que la entrevista diagnóstica ha permitido constatar que muy probablemente estamos ante un caso de MS. Ante este hecho se acuerda y planifica una cita con los padres, a la que acudirán acompañados por el niño. Antes de que ésta se produzca, se les instruye y entrena ex profeso, a fin de que lleven la iniciativa en la interacción oral con el hijo en las fases A y D. En ambas utilizan primero preguntas cerradas que solo requieren respuestas cortas (como “¿Estás a gusto?”) y luego abiertas para obtener respuestas extensas. Se enfatiza que solo han de preguntar si ven que su hijo no toma la iniciativa y en todo caso solo una vez cada dos minutos. Las preguntas se han de formular mientras que hablan entre ellos y con el hijo, tal como lo harían en la sala de espera de cualquier otra consulta.

La persona extraña para el niño es una adulta amable, que actúa como señora de la limpieza (fase Ex). Ésta permanece en la sala realizando sus labores al tiempo que interactúa oralmente con los padres e hijo, una vez que han transcurrido dos minutos desde su entrada, sin dejar de realizar sus tareas. Sólo después de una conversación con los padres se dirige al niño formulándole dos preguntas cerradas y otras dos abiertas (v.gr., “¿Me quieres decir qué es lo que no hay aquí y que a ti te gustaría que estuviera?"). Si quedase tiempo lo emplearía ordenando, repasando la limpieza y retocando los objetos, es decir, actuando pero sin hablar más hasta que se despida para irse. En este momento se inicia la situación D en la que los padres actúan como en la A, hasta llegar al final del minuto 30, momento en el que se comunica a la familia que ha surgido un imprevisto y no van a poder ser atendidos. Se les pide disculpas por la espera y se acuerda comunicarles una nueva fecha para la cita (final del tratamiento o evaluación de seguimiento).

"Entrevista para el diagnóstico de los trastornos de ansiedad en niños, versión para padres" (Anxiety Disorders Interview Schedule. Parents Interview Schedule, ADIS-IV: P; Silverman et al., 2003). Permite evaluar a través de los padres los trastornos de ansiedad y detectar otros trastornos relacionados según los criterios del DSM-IV (APA, 1994).

Dado que la ADIS-IV no incluye el MS como un trastorno de ansiedad tuvimos que verificar si los participantes cumplían los criterios del DSM-5 (APA, 2013) para su diagnóstico como trastorno primario.

Siguiendo a Bergman et al. (2008), durante la aplicación de la ADIS-IV-P estuvieron presentes dos evaluadores. Tras cada entrevista discutían sus resultados y proponían un perfil diagnóstico por consenso (criterios DSM-5; APA, 2013) que incluyó la asignación de una CSR de consenso para cada caso.

Esta entrevista, en su aplicación a los padres (Silverman, Saavedra \& Pina, 2001) ha mostrado buena fiabilidad test-retest (coeficiente kappa $=.63-.80)$ e inter-jueces $(.82 .95)$.

"Cuestionario de mutismo selectivo" (Selective Mutism Questionnaire, sMQ; Bergman, Keller, Piacentini \& Bergman, 2008). El SMQ es cumplimentado por los padres y cuantifica la frecuencia del habla del niño a través de 17 ítems agrupados en tres subescalas: hogar, escuela y situaciones sociales fuera de la escuela. Cada ítem incluye cuatro posibles respuestas $(0=$ Nunca, $1=$ Rara vez, $2=$ Frecuentemente, $3=$ Siempre $)$; a menor puntuación mayor gravedad e interferencia. Los resultados apoyan su validez convergente y discriminante (Letamendi et al., 2008), se muestra sensible a los cambios relacionados con el tratamiento (Bergman et al., 2008) y su consistencia interna es aceptable (alpha de Cronbach = .76; Bergman et al., 2013).

"Cuestionario del habla en la escuela" (School Speech Questionnaire - SSQ-; Bergman, Keller, Wood, Piacentini \& McCracken, 2001) es una medida de 6 items que cumplimentan los profesores del niño. Es una adaptación de la subescala del SMQ relativa al habla del niño en el centro educativo. Cada ítem incluye cuatro alternativas de respuestas sobre la frecuencia del habla (igual que el SMQ: a menor puntuación mayor severidad del MS y menos uso del habla). Incluye un ítem con cuatro alternativas de respuesta (No, en absoluto; 
Ligeramente; Moderadamente y Extremadamente; rango: 0-3) para valorar la interferencia de la alteración en el uso adecuado del habla en el centro educativo. No se incluye en la puntuación total.

Completamos la información diagnóstica con la obtenida mediante:

ENTREVISTA AD HOC CON LOS PADRES. Se realizaron dos entrevistas con los padres (véanse pp. 355-358 en Olivares \& Olivares-Olivares, 2018) con el fin de (i) valorar su conocimiento de la naturaleza del trastorno, (ii) conocer sus recursos para abordarlo, (iii) acotar las consecuencias que experimentaban cuando los niños tenían que enfrentarse a situaciones temidas relativas al habla y (iv) estimar la generalización del MS (lugares y las personas).

ENTREVISTA AD HOC CON LOS PROFESORES Y PERSONAL AUXILIAR DEL CENTRO EDUCATIVO. Se realizaron dos entrevistas con los profesores y el personal auxiliar (véanse pp. 355-358 en Olivares \& Olivares-Olivares, 2018) para (i) valorar el conocimiento que tenían sobre la naturaleza del trastorno, (ii) conocer su modo de afrontar el MS cuando se producía y cuando los niños tenían que enfrentarse otra vez a una situación en la que no habían hablado, (iii) valorar la generalización del MS en el centro educativo (niños, adultos y lugares).

EL INVENTARIO DE REFORZADORES INFANTILES (RSC; Cautela \& Brion-Meisels, 1979). Lo aplicamos a los padres, los profesores y el personal auxiliar, para testar los reforzadores que podríamos utilizar. Se presenta en tres formas: dos cortas (paralelas) para niños de edad entre los 3 y los 9 años y una larga (rango: 9-12 años); utilizamos las primeras. Las correlaciones momento-producto informadas por los autores en el tests-retest $(.48-.72)$ fueron todas significativas $(\mathrm{p}<.02)$.

SOCIOGRaMa. Con el fin de conocer la posición sociométrica de cada uno de los mudos selectivos, en el grupo de sus compañeros de aula, evaluamos la cohesión y la estructura de la clase en la que estaba el mudo selectivo en su centro educativo (García-Bacete \& González, 2010; González \& García-Bacete, 2010; Pérez \& Bello, 2001). Aplicamos este instrumento para disponer de índices y valores sociométricos individuales, grupales y de relación sujeto-grupo (Barrasa \& Gil, 2004), con los que construir en cada caso la prelación que utilizamos para planificar la aplicación del componente de desvanecimiento estimular que integró el tratamiento.

EVALUACIÓN DE LAS EXPECTATIVAS DE LOS COMPAÑEROS DE CLASE RESPECTO DEL HABLA DE LOS MUDOS SELECTIVos. Elaboramos un cuestionario ad hoc (Olivares \& Olivares-Olivares, 2008 ), de aplicación individual, con dos ítems y tres alternativas de respuesta (mucho, poco o nada). En el primero preguntábamos “¿Qué pasaría si una persona mayor se dirigiese a $\mathrm{X}$ —nombre del mudo selectivo - para hacerle una pregunta? ¿Le contestaría X hablándole mucho, hablándole poco o no hablándole nada?” En el segundo se preguntaba “¿Y si le preguntaras tú a X? ¿Te respondería hablándote mucho, hablándote poco o no hablándote nada?" Esta estrategia también la utilizamos tras la reproducción de la grabación audiovisual en la que se veía y oía hablar al mudo selectivo con sus compañeros de actividad cooperativa.

La intervención psicológica fue valorada por padres y profesores, una vez concluida, cumplimentando el "Cuestionario de satisfacción del cliente" (Client Satisfaction Questionnaire - CSQ-; Hargreaves \& Attkisson, 1978). Se trata de una medida general, de ocho ítems. Cada ítem contiene cuatro opciones de respuesta (rango: 1-4) donde 1 = Mala y 4 = Excelente; a mayor puntuación total mayor satisfacción del cliente (rango: 8-32). Los ítems 2, 4, 5 y 8 tienen puntuación inversa. El CSQ ha mostrado tener buenas propiedades psicométricas, incluyendo una excelente consistencia interna (.91) e indicios significativos respecto del uso del servicio y el resultado de la intervención (Attkisson \& Zwick, 1982). Tanto en su aplicación con los padres como con los profesores modificamos la redacción inicial de cada ítem con expresiones como "Su hijo..." o "Su alumno..." manteniendo igual el resto de su contenido.

\section{Procedimiento}

Iniciamos el proceso con dos encuentros con los padres. En el primero se aplicó la ADIS-IV-P y un cuestionario con los criterios diagnósticos para el MS contenidos en el DSM-5. En la segunda les dimos los resultados del test situacional y de la ADIS-IV-P, pidiéndoles que cumplimentaran el SMQ. Asimismo, se les informó, instruyó y entrenó para actuar durante la aplicación del EHEx.

Confirmada la existencia del MS se realizaron las entrevistas con el personal del centro educativo implicado, la dirección y la jefatura de estudios, completando la evaluación aplicando el sociograma, el inventario de reforzadores (RSC) y evaluando las expectativas de los compañeros de aula sobre el habla de los mudos selectivos. Los profesores cumplimentaron el ssQ. 
Todas las pruebas y la ADIS-IV-P se aplicaron en los momentos de la evaluación (antes de iniciar el tratamiento, al terminarlo y en la medida de seguimiento). En todas las aplicaciones del EHEx "la señora de la limpieza" actuó de acuerdo con el protocolo, pero fue distinta en cada medida para cumplir con la condición de ser extraña para los niños.

\section{Diseño}

Utilizamos un diseño de acumulación de casos (Ato, López \& Benavente, 2013), con medidas antes del tratamiento, tras cumplirse el criterio para terminarlo y en un seguimiento a los 12 meses.

\section{Tratamiento}

Su integridad se controló con un manual (Olivares \& Olivares-Olivares, 2008b). Este contempla que, como paso previo a su aplicación, se contacte con la asociación de madres y padres de alumnos (AMPA), la dirección y el consejo escolar, con el profesor y personal auxiliar que participa en la educación del mudo selectivo, así como con la inspección educativa si lo estimara conveniente la dirección del centro a fin de justificar la necesidad de aplicarlo en el contexto educativo.

A todos se les indica que el tratamiento incluye el desvanecimiento estimular y ello implica que el profesor del mudo selectivo, que es el único al que todavía habla en el centro (al menos en alguna ocasión), tiene que tener un papel central en la primera parte del tratamiento. Asimismo, es necesaria la autorización e implicación de los órganos y autoridades del centro para que permitan y faciliten las modificaciones de la actividad docente, gestionen las autorizaciones escritas de los padres y financien los costes de la compra del material del juego cooperativo (y el viaje fin de curso o cualquier otra actividad significativa para toda la clase que pueda motivar la implicación de la mayoría de los alumnos en la actividad cooperativa).

Antes de iniciar el tratamiento todos han de dar su conformidad escrita o firmar un consentimiento colectivo informado.

\section{Tratamiento de padres, profesores y personal auxiliar}

Comienza con la fase psicoeducativa, destinada a transmitir información relativa al MS y explicar su naturaleza. Utilizamos un manual para padres y profesores ("El niño con miedo a hablar"; Olivares, 1994) y un modelo tentativo (Olivares-Olivares \& Olivares, 2018), a lo que se añade una descripción de la estructura, objetivos, contenidos y actividades previstas para padres, profesores y personal auxiliar que interactúa cotidianamente con el mudo selectivo.

Esta fase se desarrolla durante una semana e incluye dos sesiones de 90 minutos, para los padres y otras dos para los profesionales, incluyendo un descanso de 15 minutos por sesión. Durante éstas se resuelven las dudas, se fija un horario de atención para resolver nuevas dudas y recoger sugerencias de mejora, se facilita un número de teléfono móvil y una dirección de correo electrónico para realizarlas y para solicitar una cita presencial con los responsables del tratamiento. En nuestro caso fueron los lunes, los miércoles y los viernes lectivos, de 18 a 19 horas. Los mensajes también se contestaron esos días.

La segunda y la tercera fase son las de entrenamiento y las tareas para casa, respectivamente. En la primera los padres y el personal del centro son instruidos y entrenados en dos sesiones de 90 minutos (15 minutos descanso/sesión), una por semana, incluyendo al final las "tareas para casa". La segunda sesión se inicia con la puesta en común de los logros/dificultades en la realización de las tareas acordadas y continúa con el análisis de las relaciones entre los antecedentes, las respuestas y los consecuentes ( $\mathrm{ABC}$ ), la explicación cognitivo-conductual, la transmisión de nueva información y el modelado de cómo prevenir/afrontar tales dificultades y los imprevistos.

El entrenamiento del personal del centro educativo se realiza con ejemplos específicos extraídos de lo que acontece cuando los niños tienen que hablar y no lo hacen.

Los componentes del tratamiento de los adultos son la transmisión de información y la reestructuración cognitiva (explicación, operacionalización de conceptos y descripción de situaciones problemáticas en términos $\mathrm{ABC})$, las instrucciones verbales y el modelado sobre cómo actuar adecuadamente. 


\section{Tratamiento de los niños}

Se limita a la fase de entrenamiento. La duración de la sesión es de 60 minutos y su periodicidad diaria (días lectivos). Su aplicación se desarrolla en dos partes cuyos objetivos generales y específicos, el criterio de logro y los componentes del entrenamiento pueden verse en la Tabla 1.

En las dos partes se utiliza una tarea mediadora, consistente en un juego cooperativo como, por ejemplo, la confección de rompecabezas de dificultad elevada para la edad de los niños (Olivares-Olivares, Macià \& Olivares, 2019) o el dominó de animales (Balbuena-Teruel, Rueda-Lozano \& López-Herrero, 2012). El fin es disponer de una tarea que requiera el uso del habla audible y permita incorporar nuevos jugadores, siguiendo la prelación establecida para la aplicación del desvanecimiento estimular a partir de los resultados del test sociométrico, así como una implicación oral cada vez más activa del mudo selectivo. De ser posible, esta tarea se ha de seleccionar a partir de los resultados del inventario de reforzadores de actividad (RSC; Cautela \& BrionMeisels, 1979).

Tabla 2. Objetivos, componentes del entrenamiento del mudo selectivo y registro de la fecha en la que se alcanza el criterio de logro

\begin{tabular}{|c|c|c|c|c|}
\hline \multirow{6}{*}{ : } & $\begin{array}{l}\text { OBJETIVO GENERAL: desencadenar y generalizar } \\
\text { el uso adecuado del habla audible en un grupo de } \\
\text { cuatro integrantes y sus profesoras mientras se rea- } \\
\text { liza un juego cooperativo (dominó de nombres de } \\
\text { animales, composición de rompebezas, etc.) }\end{array}$ & $\begin{array}{l}\text { Registro } \\
\text { del logro }{ }^{\mathrm{a}, \mathrm{b}}\end{array}$ & $\begin{array}{l}\text { Componentes del } \\
\text { entrenamiento }\end{array}$ & \\
\hline & OBJETIVOS ESPECÍFICOS & & & \multirow{5}{*}{ 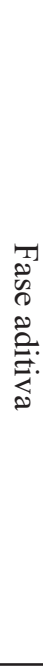 } \\
\hline & 1. Responder al profesor estando a solas & & $\begin{array}{l}\text { Desvanecimiento. Refuerzo } \\
\text { social y de actividad. }\end{array}$ & \\
\hline & $\begin{array}{l}\text { 2. Responder al profesor y al primer compañero } \\
\text { incorporado, en presencia de ambos }\end{array}$ & & $\begin{array}{l}\text { Desvanecimiento. Refuerzo } \\
\text { social y de actividad. }\end{array}$ & \\
\hline & $\begin{array}{l}\text { 3. Argumentar oralmente las razones de la activi- } \\
\text { dad que se desea realizar, en su turno de partici- } \\
\text { pación en el juego cooperativo, en presencia de su } \\
\text { profesora y de los dos compañeros incorporados }\end{array}$ & & $\begin{array}{l}\text { Desvanecimiento. Refuerzo } \\
\text { social y de actividad. } \\
\text { Modelado. Economía de } \\
\text { fichas. Coste de respuesta. } \\
\text { Presión de grupo. }\end{array}$ & \\
\hline & $\begin{array}{l}\text { 4. Hablar a los tres compañeros de grupo de juego } \\
\text { cooperativo durante la realización de éste. }\end{array}$ & & $\begin{array}{l}\text { Desvanecimiento. Refuerzo } \\
\text { social y de actividad. } \\
\text { Economía de fichas. Coste } \\
\text { de respuesta. Presión de } \\
\text { grupo. }\end{array}$ & \\
\hline \multirow{4}{*}{ 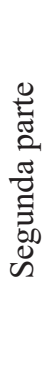 } & $\begin{array}{l}\text { OBJETIVO GENERAL: Eliminar el aislamiento social } \\
\text { y consolidar y generalizar el uso del habla }\end{array}$ & & \multirow{4}{*}{$\begin{array}{l}\text { Desvanecimiento. } \\
\text { Exposición. } \\
\text { Refuerzo social y de } \\
\text { actividad. Presión de } \\
\text { grupo. }\end{array}$} & \multirow{4}{*}{ 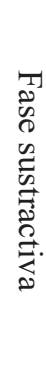 } \\
\hline & OBJETIVOS ESPECÍFICOS & & & \\
\hline & $\begin{array}{l}\text { 5. Exponer al mudo selectivo a los efectos de la } \\
\text { visualización, por sus grupo-clase, del vídeo en el } \\
\text { que aparece hablando con otros compañeros }\end{array}$ & & & \\
\hline & $\begin{array}{l}\text { 6. Generalizar y consolidar el uso del habla en } \\
\text { el aula }\end{array}$ & & & \\
\hline
\end{tabular}

Notas: (a) $80 \%$ de éxito en el uso adecuado del habla audible en dos días consecutivos de actividad registrada. (b) Fecha en la que se alcanza el criterio requerido para la superación del objetivo específico. 


\section{PRIMERA PARTE}

Seleccionada la tarea cooperativa más adecuada para el caso, la primera parte del entrenamiento se planifica según la secuencia de objetivos que se recogen en la Tabla 1.

La incorporación de los participantes durante el proceso de desvanecimiento se realizó solicitándoles que se quedaran para ayudar a su profesora. No se argumentó nada más. Los niños no sabían que se quedarían, pero sí sus padres a quienes se les solicitó previamente permiso escrito (a través del consejo escolar y la AMPA).

En la incorporación de cada niño sus tres primeras posiciones en la sala de la clase son, primero, sentarse de espaldas al mudo selectivo (en el otro extremo de la diagonal del lugar donde se hallaba), después de perfil y por último mirando a la mesa del profesor donde se hallaba el mudo selectivo realizando sus tareas. Cada uno de los cambios restantes del incorporado, durante la progresión de las aproximaciones en la sala hasta quedar sentado frente al niño con MS, se producen tras alcanzar el criterio de logro en dos sesiones consecutivas (responder oralmente y de manera audible en el $80 \%$ de ocasiones en cada una).

Los cambios se producen a instancias del profesor, una vez que es informado de los resultados del registro obtenidos tras visualizar por las tardes, dos observadores independientes, la ocurrencia del habla audible en los vídeos grabados por las mañanas durante el desarrollo del juego cooperativo.

Asimismo, al profesor se le instruye y entrena para combinar sus tareas (planificar actividades, preparar materiales, corregir, etc.) con la interacción oral con los niños, principalmente con el mudo selectivo mientras realizan las actividades que requiere el juego, pero estando alerta para reducir su frecuencia en la medida en que se incrementa la intensidad del volumen del habla del mudo selectivo.

De igual modo, cada cinco minutos, le tiene que formular al menos una pregunta que requiera su respuesta oral. En el inicio de la aplicación del desvanecimiento, y tras cada incorporación, las preguntas han de ser cerradas, pero cuando éstas comienzan a producirse se incluyen también las abiertas. El profesor registra el tipo y la frecuencia de sus preguntas para orientarse respecto del número y la categoría de las que ha realizado.

Para el logro del primer objetivo específico el profesor y el mudo selectivo realizan las actividades del juego cooperativo hasta alcanzar el criterio de logro fijado. Superado éste, al iniciar la actividad para lograr el segundo, se da a conocer al mudo selectivo y al niño recién incorporado que hay una norma que exige que la tarea la han de realizar solicitándose la colaboración de manera audible (por ejemplo, durante la construcción de un rompecabezas se pide a quien te precede en el juego su opinión sobre la pieza que se quiere colocar).

En relación con el tiempo disponible, para realizar la actividad que le corresponde a cada uno en su turno de actuación, se indica a los participantes que cada uno dispone de dos minutos para decidir lo que desea hacer y hacerlo. Si tarda más entonces se le penaliza con la pérdida de un punto por minuto/fracción. Se pone a disposición de los niños un cronómetro que cada uno pone en marcha/detiene cuando le toca actuar/ha actuado.

Al incorporarse el segundo participante (inicio de la actividad propia del tercer objetivo) el profesor les indica que, además de la petición razonada de la ayuda a quien le precede (el lugar donde se sientan se asigna aleatoriamente en cada sesión), también hay que justificar con voz audible por qué se toma la decisión para actuar como se hace. Para facilitarles la argumentación se les informa que las razones deben centrarse en aspectos concretos de la actividad (v.gr., en el rompecabezas la forma de la pieza, la continuidad del dibujo o del color entre la pieza y las del lugar donde la iba a colocar). El profesor pone tres ejemplos modelando tres modos distintos de argumentar por qué e indica que a partir de ese instante los participantes van a obtener beneficios por realizar la actividad cooperativa, pero cumpliendo nuevas reglas. Éstas son:

- Los puntos ganados por cada participante van a un fondo común (se enfatiza la rentabilidad de la colaboración). El montante de éste pasa a ser cada dos días propiedad de uno de ellos, siguiendo un orden que se establece por sorteo. Cada uno puede gastar los puntos de su cuenta el día siguiente de obtenerlos o acumularlos hasta un máximo de 30 (disfrute de un "recreo extra" completo).

- El dúo (y posteriormente el trío y el cuarteto — con las nuevas incorporaciones - ) dispone de tres intentos consecutivos para argumentar y realizar su aportación al juego, manteniéndose la regla vigente para la actuación individual (dos minutos para realizar su aportación y penalización con la pérdida de un punto por minuto/fracción por excederse). Si lo consiguen entonces reciben un punto (un minuto de "recreo extra"). Tras el tercer fallo consecutivo se resta un punto de la cuenta común. 
- El gasto de todos o parte de los puntos acumulados está vinculado a compartirlo con uno o dos niños de la clase (incluidos los del grupo de actividad cooperativa), que han de ser distintos cada vez.

Al iniciarse la aplicación de la economía de fichas, durante el desarrollo de la actividad del tercer objetivo, el profesor entrega gratuitamente tres puntos a cada participante.

Para información y (auto)control de los participantes se fijan en la pared de la clase dos carteles de 2 metros de largo por 1,5 de ancho, en ambos laterales y a una altura de 1,5 metros. Uno contiene las reglas y las normas de canje; el otro, dos tablas de doble entrada. En este último, una tabla contendrá en su primera columna los nombres de los participantes (acompañados de sus fotos) y en la primera fila los días lectivos del mes; en las intersecciones (nombre del niño y día de la semana) se anotan los puntos que diariamente cada uno aporta al fondo; la otra contendrá, en la intersección de los nombres (primera columna) y los días lectivos (primera fila), los puntos sin gastar y gastados en cada "cuenta particular" (nombre x día lectivo).

Cuando se incorpora el tercer niño al juego cooperativo se inicia la actividad para el logro del cuarto objetivo. Mientras desarrollan su actividad los cuatro niños, el profesor les sigue manifestando a todos y a cada uno su admiración por el modo como la están haciendo (reconociendo y alabando algo de cada uno y especialmente del mudo selectivo). Una vez superado el criterio de logro, les pedirá permiso para grabarlos mientras realizan la tarea. Les indica que la decisión la tienen que tomar por mayoría (tres de cuatro) y que, si optan por autorizarlo, regalará a cada uno un recreo extra completo (compartido con al menos un compañero). Justifica su petición indicándoles que desea que los compañeros de la clase los puedan ver cómo resuelven las dificultades que les plantea el juego cooperativo porque quiere organizar un concurso en el que participe toda la clase.

El profesor debe estimular y dejar que los cuatro discutan los pros y contras para tomar su decisión por mayoría. La variable "presión de grupo" debe hacer su trabajo. Una vez autorizado, durante dos sesiones actuará como si estuviese grabando la actividad del grupo, pero ésta se realizará realmente dos días después. Del material audiovisual grabado se seleccionan 15 minutos. La grabación ha de dedicar el 50\% del contenido al mudo selectivo y a mostrar cómo se apasionan e irritan (si ocurre) durante la realización de juego cooperativo, sobre todo cuando están en la tercera elección.

\section{SEGUNDA PARTE}

En ésta la actividad se orienta al logro de los dos últimos objetivos específicos.

Cuando se tenga disponible la grabación audiovisual, media hora antes del recreo del siguiente día lectivo, el profesor, con el mudo selectivo presente, la reproduce para que todos los alumnos de la clase puedan ver a su compañero con MS hablando, participando y discutiendo con los tres compañeros de su grupo de juego.

Ese mismo día, media hora antes del final de la clase, el profesor se dirigirá a sus alumnos para indicarles que pretende organizar un concurso, que durará cinco días lectivos, para competir por equipos en la realización del juego cooperativo que han visto que realizaban sus compañeros. Les indicará que todos los que quieran participar tienen que traer el día siguiente una autorización paterna para quedarse en el centro una hora cada día, para realizar la actividad. Asimismo, les informará que el grupo vencedor elegirá, a partir de una relación que les presentará, por ejemplo, el lugar de destino del viaje de fin de curso de entre las tres propuestas más votadas por todos.

Se recomienda que los grupos de trabajo estén integrados por cuatro participantes para maximizar la frecuencia de la participación de cada uno en la actividad cooperativa.

Cuando se disponga de las autorizaciones el profesor confeccionará y dará a conocer los grupos de trabajo e informará de las normas que regirán durante el desarrollo de la actividad (las mismas que ya se han aplicado en el grupo del mudo selectivo). Después, los miembros de los grupos elegirán cada uno a su coordinador, por votación entre ellos. En esta investigación el mudo selectivo siempre coordinó su grupo.

Entre las funciones de los coordinadores está la de mediar cuando haya conflicto en la toma de decisiones para resolver los problemas que plantee el juego cooperativo; deben sentenciar quién tiene razón. Asimismo, han de garantizar que se mantenga el orden acordado en la participación, llamar a cada miembro del grupo por su nombre para darle la vez, vigilar el cumplimiento de las normas que regulan la actividad, controlar el tiempo del que dispone cada participante (vigilar y ordenar en su caso la puesta en marcha/paro del cronómetro), consultar con otros grupos si se presenta la circunstancia de que no hubiera unanimidad en el suyo sobre 
la actividad que se debe realizar cuando se esté en el tercer intento de solucionar cada problema que pueda plantearse durante el juego, etc. El objetivo es maximizar las consultas entre los coordinadores de los grupos. El papel del profesor es observar el trabajo de los grupos y colaborar con los coordinadores en la vigilancia del cumplimiento de las normas.

Si ningún grupo hubiera logrado terminar antes el juego cooperativo, entonces, transcurridos los cinco días de competición ésta se da por terminada y se valora el progreso de cada grupo en la tarea para determinar el ganador. Al día siguiente un miembro del AMPA, el director del centro y el profesor del aula proclaman el grupo vencedor y éste decide mediante votación entre sus miembros, por ejemplo, el lugar de destino para el viaje fin de curso.

\section{Análisis de datos}

Se aplicó la prueba $T$ de comparación para muestras relacionadas y se calculó el índice de tamaño del efecto a partir de la $d$ de Cohen (Cohen, 1988). Se estudió la diferencia en la comorbilidad antes y después del tratamiento, y a los 12 meses de seguimiento mediante la prueba no paramétrica $Q$ de Cochran. No se produjeron casos perdidos; todos los participantes fueron incluidos en el análisis de datos que se realizó con el paquete estadístico SPSS 25 (IBM, 2017).

\section{Resultados}

El promedio del coeficiente kappa interobservadores para el EHEx fue de .90 y el de la CSR de .93 . Las medias y desviaciones típicas del número de las semanas y de las sesiones de tratamiento (una por día lectivo) fueron respectivamente:15.18 $(D T=3.67)$ y $75.90(D T=18.32)$.

\section{Resultados de la severidad clínica}

La Tabla 3 presenta las pruebas $\mathrm{T}$ para muestras relacionadas en las diferentes subescalas del SMQ informado tanto por padres como por profesores. Se puede observar que existen diferencias significativas entre los diferentes momentos temporales, mejorando no sólo en el postest sino incrementando las mejoras en el seguimiento. Los tamaños del efecto son muy altos, alcanzando la mayor magnitud del pretest al seguimiento. La severidad clínica presentó diferencias estadísticamente significativas del pretest al postest $\left(T_{(9)}=22.04 ; p<.001\right)$, del pretest al seguimiento $\left(T_{(9)}=24.60 ; p<.001\right) \mathrm{y}$ del postest al seguimiento $\left(T_{(9)}=8.14 ; p<.001\right)$, disminuyendo tras el tratamiento y en el seguimiento. 
Tabla 3. Pruebas Ty tamaños del efecto en el $S M Q$

\begin{tabular}{|c|c|c|c|c|}
\hline $\begin{array}{l}\text { Variable } \\
\text { dependiente }\end{array}$ & $\begin{array}{l}\text { Momento } \\
\text { Temporal } \\
\end{array}$ & $\mathrm{M} \pm \mathrm{DT}$ & $\mathrm{T}$ & $\mathrm{d}_{+}$ \\
\hline $\begin{array}{l}\text { Mutismo en la } \\
\text { escuela }^{1}\end{array}$ & $\begin{array}{l}\text { Pretest } \\
\text { Postest } \\
\text { Seguimiento }\end{array}$ & $\begin{array}{l}3.70 \pm 1.88 \\
12.60 \pm 084 \\
15.20 \pm 0.78\end{array}$ & $\begin{array}{l}\text { Pre-post: } T_{(9):}-19.42 ; p<.001 \\
\text { Pre-seg: } T_{(9)}=-17.58 ; p<.001 \\
\text { Post-seg: } T_{(9)}=-6.09 ; p<.001\end{array}$ & $\begin{array}{l}4.32 \\
5.59 \\
2.83\end{array}$ \\
\hline $\begin{array}{l}\text { Mutismo en } \\
\text { las situaciones } \\
\text { familiares }^{1}\end{array}$ & $\begin{array}{l}\text { Pretest } \\
\text { Postest } \\
\text { Seguimiento }\end{array}$ & $\begin{array}{l}7.20 \pm 2.09 \\
12.40 \pm 069 \\
14.90 \pm 0.74\end{array}$ & $\begin{array}{l}\text { Pre-post: } T_{(9)}:-9.93 ; p<.001 \\
\text { Pre-seg: } T_{(9)}=-16.29 ; p<.001 \\
\text { Post-seg: } T_{(9)}=-11.18 ; p<.001\end{array}$ & $\begin{array}{l}2.27 \\
3.37 \\
3.31\end{array}$ \\
\hline $\begin{array}{l}\text { Mutismo } \\
\text { Contexto social } \\
\text { no escolar }\end{array}$ & $\begin{array}{l}\text { Pretest } \\
\text { Postest } \\
\text { Seguimiento }\end{array}$ & $\begin{array}{l}1.00 \pm 1.04 \\
9.50 \pm 1.43 \\
11.70 \pm 0.67\end{array}$ & $\begin{array}{l}\text { Pre-post: } T_{(9)}:-31.62 ; p<.001 \\
\text { Pre-seg: } T_{(9)}=-27.03 ; p<.001 \\
\text { Post-seg: } T_{(9)}=-4.71 ; p=.001\end{array}$ & $\begin{array}{l}7.47 \\
9.40 \\
1.54\end{array}$ \\
\hline $\begin{array}{l}\text { Puntuaciòn } \\
\text { total mutismo } \\
\text { selectivo }^{1}\end{array}$ & $\begin{array}{l}\text { Postest } \\
\text { Postest } \\
\text { Seguimiento }\end{array}$ & $\begin{array}{l}11.90 \pm 4.63 \\
33.20 \pm 2.66 \\
41.90 \pm 099\end{array}$ & $\begin{array}{l}\text { Pre-post: } T_{(9)=}=-29.76 ; p<.001 \\
\text { Pre-seg: } T_{(9)}^{=-24.40 ; p<.001} \\
\text { Post-seg: } T_{(9)}=-14.13 ; p<.001\end{array}$ & $\begin{array}{l}4.20 \\
5.92 \\
2.99\end{array}$ \\
\hline $\begin{array}{l}\text { Mutismo en la } \\
\text { escuela }^{2}\end{array}$ & $\begin{array}{l}\text { Pretest } \\
\text { Postest } \\
\text { Seguimiento }\end{array}$ & $\begin{array}{l}2.70 \pm 2.11 \\
11.70 \pm 0.67 \\
13.10 \pm 0.99\end{array}$ & $\begin{array}{l}\text { Pre-post: } T_{(9)}=-12.87 ; p<.001 \\
\text { Pre-seg: } T_{(9)}=-23.00 ; p<.001 \\
\text { Post-seg: } T_{(9)}=-4.58 ; p=.001\end{array}$ & $\begin{array}{l}3.90 \\
4.50 \\
1.91\end{array}$ \\
\hline
\end{tabular}

${ }^{1}$ Informado por los padres ${ }^{2}$ Informado por los profesores

$\mathrm{SMQ}=$ Selective Mutism Questionnaire $\quad M=$ Media $\quad D T=$ Desviación típica $\quad T=$ prueba para muestras relacionadas $\quad d+=$ Tamaño del efecto (valor de la magnitud de las comparaciones pretest-postest, pretest-seguimiento, postest-seguimiento).

\section{Resultados de la interferencia y el malestar}

Los resultados de la interferencia del MS, informado por los padres, indican que ésta disminuye tras la aplicación del tratamiento en todas las áreas (escolar, familiar, extraescolar y total). Además, la interferencia en la escuela, informada por los profesores, también decrece tras el tratamiento y en seguimiento a los 12 meses. El malestar/sufrimiento informado por los niños y sus padres decreció en el postest y siguió haciéndolo en el seguimiento (véase la Tabla 4). 
Tabla 4. Pruebas $T$ y tamaños del efecto en interferencia y malestar

\begin{tabular}{|c|c|c|c|c|}
\hline Variable dependiente & $\begin{array}{l}\text { Momento } \\
\text { Temporal }\end{array}$ & $M \pm D T$ & $T$ & $d_{+}$ \\
\hline $\begin{array}{l}\text { Interferencia en la } \\
\text { escuela }\end{array}$ & $\begin{array}{c}\text { Pretest } \\
\text { Postest } \\
\text { Seguimiento }\end{array}$ & $\begin{array}{l}2.10 \pm 0.87 \\
1.00 \pm 0.00 \\
0.50 \pm 0.53\end{array}$ & $\begin{array}{l}\text { Pre-post: } T_{(9)}: 3.97 ; p=.003 \\
\text { Pre-seg: } T_{(9)}=5.24 ; p=.001 \\
\text { Post-seg: } T_{(9)}=3.00 ; p=.015\end{array}$ & $\begin{array}{l}1.16 \\
1.68 \\
0.46\end{array}$ \\
\hline Interferencia en la familia & $\begin{array}{l}\text { Pretest } \\
\text { Postest } \\
\text { Seguimiento }\end{array}$ & $\begin{array}{l}1.50 \pm 0.71 \\
0.80 \pm 0.42 \\
0.30 \pm 0.48\end{array}$ & $\begin{array}{l}\text { Pre-post: } T_{(9)}=3.28 ; p=.010 \\
\text { Pre-seg: } T_{(9)}=9.00 ; p<.001 \\
\text { Post-seg: } \mathrm{T}_{(9)}=3.00 ; p=.015\end{array}$ & $\begin{array}{l}0.90 \\
1.54 \\
1.09\end{array}$ \\
\hline $\begin{array}{l}\text { Interferencia contexto } \\
\text { extraescolar }\end{array}$ & $\begin{array}{l}\text { Pretest } \\
\text { Postest } \\
\text { Seguimiento }\end{array}$ & $\begin{array}{l}2.80 \pm 0.42 \\
1.70 \pm 0.48 \\
1.00 \pm 0.00\end{array}$ & $\begin{array}{l}\text { Pre-post: } T_{(9)}=6.13 ; p<.001 \\
\text { Pre-seg: } T_{(9)}^{(9)}=13.50 ; p<.001 \\
\text { Post-seg: } T_{(9)}=4.58 ; p=.001\end{array}$ & $\begin{array}{l}2.39 \\
3.92 \\
1.33\end{array}$ \\
\hline $\begin{array}{l}\text { Interferencia total } \\
\text { Escuela+Familia } \\
+ \text { Situaciones sociales } \\
\text { extraesc. }\end{array}$ & $\begin{array}{l}\text { Postest } \\
\text { Postest } \\
\text { Seguimiento }\end{array}$ & $\begin{array}{l}6.40 \pm 1.65 \\
3.70 \pm 0.48 \\
1.80 \pm 0.79\end{array}$ & $\begin{array}{c}\text { Pre-post: } T_{(9)}: 6.02 ; p<.001 \\
\text { Pre-seg: } T_{(9)}=10.77 ; p<.001 \\
\text { Post-seg: } T_{(9)}=6.86 ; p<.001\end{array}$ & $\begin{array}{l}1.50 \\
2.55 \\
3.62\end{array}$ \\
\hline $\begin{array}{l}\text { Interferencia total } \\
\text { evaluada por los padres }\end{array}$ & $\begin{array}{l}\text { Postest } \\
\text { Postest } \\
\text { Seguimiento }\end{array}$ & $\begin{array}{c}2.10 \pm .32 \\
0.50 \pm 0.53 \\
1.00 \pm 0.00\end{array}$ & $\begin{aligned} & \text { Pre-post: } T_{(9)}=11.00 ; p<.001 \\
& \text { Pre-seg: } T_{(9)}=9.79 ; p<.001 \\
& \text { Post-seg: } T_{(9)}^{(9)}=3.00 ; p=.015\end{aligned}$ & $\begin{array}{c}4.57 \\
3.14 \\
-0.86\end{array}$ \\
\hline $\begin{array}{l}\text { Interferencia en la } \\
\text { escuela evaluada por los } \\
\text { profesores }\end{array}$ & $\begin{array}{l}\text { Pretest } \\
\text { Postest } \\
\text { Seguimiento }\end{array}$ & $\begin{array}{l}2.30 \pm 0.82 \\
1.00 \pm 0.00 \\
0.60 \pm 0.52\end{array}$ & $\begin{array}{l}\text { Pre-post: } T_{(9)}=4.99 ; p=.001 \\
\text { Pre-seg: } T_{(9)}^{(9)}=6.53 ; p<.001 \\
\text { Post-seg: } T_{(9)}=2.45 ; p=.037\end{array}$ & $\begin{array}{l}0.99 \\
1.89 \\
0.37\end{array}$ \\
\hline $\begin{array}{l}\text { Malestar observado en el } \\
\text { niño por los padres }\end{array}$ & $\begin{array}{l}\text { Pretest } \\
\text { Postest } \\
\text { Seguimiento }\end{array}$ & $\begin{array}{l}2.20 \pm 0.63 \\
1.00 \pm 0.00 \\
0.40 \pm 0.52\end{array}$ & $\begin{array}{l}\text { Pre-post: } T_{(9)}=6.00 ; p<.001 \\
\text { Pre-seg: } T_{(9)}=7.22 ; p<.001 \\
\text { Post-seg: } T_{(9)}=3.67 ; p=.005\end{array}$ & $\begin{array}{l}1.74 \\
2.61 \\
0.87\end{array}$ \\
\hline $\begin{array}{l}\text { Malestar experimentado } \\
\text { w informado por los } \\
\text { padres }\end{array}$ & $\begin{array}{c}\text { Pretest } \\
\text { Postest } \\
\text { Seguimiento }\end{array}$ & $\begin{array}{l}2.60 \pm 0.52 \\
1.10 \pm 0.32 \\
0.30 \pm 0.48\end{array}$ & $\begin{array}{l}\text { Pre-post: } T_{(9)}=9.00 ; p<.001 \\
\text { Pre-seg: } T_{(9)}=15.06 ; p<.001 \\
\text { Post-seg: } T_{(9)}=6.00 ; p=.001\end{array}$ & $\begin{array}{l}2.64 \\
4.04 \\
2.29\end{array}$ \\
\hline
\end{tabular}

$M=$ Media $D T=$ Desviación típica $T=$ Prueba para muestras relacionadas $d+=$ Tamaño del efecto

\section{Resultados en el test situacional}

En la Tabla 5 se presentan los resultados de las variables dependientes registradas en el EHEx. Se puede observar que los niños mejoran del pretest al postest, aumentando los logros en el seguimiento en todas las variables excepto en las registradas entre padres e hijo en presencia del extraño. Los tamaños del efecto confirman los resultados, siendo los más altos, en la mayoría de las variables, los alcanzados entre prestest y seguimiento.

Tabla 5. Pruebas Ty tamaños del efecto en el EHEx

\begin{tabular}{ccccc}
\hline Variable dependiente & $\begin{array}{c}\text { Momento } \\
\text { Temporal }\end{array}$ & $M \pm D T$ & $T$ & $d_{+}$ \\
\hline \multicolumn{5}{c}{ Interacción Niño y padres solos } \\
\hline \multirow{3}{*}{ Habla no audible } & Pretest & $1.40 \pm 1.17$ & Pre-post: $T_{(9)}=0.426 ; p=.678$ & 0.08 \\
& Postest & $1.30 \pm 0.67$ & Pre-seg: $T_{(9)}=3.72 ; p=.004$ & 1.09 \\
& Seguimiento & $0.00 \pm 0.00$ & Post-seg: $T_{(9)}=6.09 ; p<.001$ & 1.77 \\
Habla audible & Pretest & $0.00 \pm 0.00$ & Pre-post: $\mathrm{T}(9)=-20.12 ; \mathrm{p}<.001$ & 4.11 \\
& Postest & $4.50 \pm 0.71$ & Pre-seg: $\mathrm{T}(9)=-49.00 ; \mathrm{p}<.001$ & 4.48 \\
& Seguimiento & $4.90 \pm 0.32$ & Post-seg: $\mathrm{T}(9)=-1.81 ; \mathrm{p}=.104$ & 0.51
\end{tabular}




\begin{tabular}{|c|c|c|c|c|}
\hline $\begin{array}{l}\text { Habla audible } \\
\text { espontánea }\end{array}$ & $\begin{array}{c}\text { Pretest } \\
\text { Postest } \\
\text { Seguimiento }\end{array}$ & $\begin{array}{l}0.00 \pm 0.00 \\
1.90 \pm 0.74 \\
2.80 \pm 0.79\end{array}$ & $\begin{array}{l}\text { Pre-post: } T_{(9)}=-8.14 ; p<.001 \\
\text { Pre-seg: } T_{(9)}=-11.22 ; p<.001 \\
\text { Post-seg: } T_{(9)}^{(}=-5.01 ; p=.001\end{array}$ & $\begin{array}{l}1.74 \\
2.56 \\
1.11\end{array}$ \\
\hline Frecuencia del habla & $\begin{array}{c}\text { Postest } \\
\text { Postest } \\
\text { Seguimiento }\end{array}$ & $\begin{array}{c}3.50 \pm 3.77 \\
20.90 \pm 4.43 \\
26.90 \pm 3.45\end{array}$ & $\begin{array}{l}\text { Pre-post: } T_{(9)}=-15.04 ; p<.001 \\
\text { Pre-seg: } T_{(9)}=-19.29 ; p<.001 \\
\text { Post-seg: } T_{(9)}=-28.76 ; p<.001\end{array}$ & $\begin{array}{l}4.22 \\
5.67 \\
1.24\end{array}$ \\
\hline \multicolumn{5}{|c|}{ Interacción Niño y padres en presencia de un extraño } \\
\hline Habla no audible & $\begin{array}{l}\text { Postest } \\
\text { Postest } \\
\text { Seguimiento }\end{array}$ & $\begin{array}{l}0.20 \pm 0.42 \\
1.10 \pm 0.57 \\
1.10 \pm 0.57\end{array}$ & $\begin{array}{l}\text { Pre-post: } T_{(9)}=-3.86 ; p=.004 \\
\text { Pre-seg: } T_{(9)}=-3.86 ; p=.004\end{array}$ & $\begin{array}{l}1.96 \\
1.96 \\
0.00\end{array}$ \\
\hline Habla audible & $\begin{array}{l}\text { Pretest } \\
\text { Postest } \\
\text { Seguimiento }\end{array}$ & $\begin{array}{l}0.00 \pm 0.00 \\
4.00 \pm 0.94 \\
4.00 \pm 0.94\end{array}$ & $\begin{array}{l}\text { Pre-post: } T_{(9)}=-13.42 ; p<.001 \\
\text { Pre-seg: } T_{(9)}=-13.42 ; p<.001\end{array}$ & $\begin{array}{l}3.66 \\
3.66 \\
0.00\end{array}$ \\
\hline $\begin{array}{l}\text { Habla audible } \\
\text { espontánea }\end{array}$ & $\begin{array}{l}\text { Pretest } \\
\text { Postest } \\
\text { Seguimiento }\end{array}$ & $\begin{array}{l}0.00 \pm 0.00 \\
1.20 \pm 0.42 \\
1.20 \pm 0.42\end{array}$ & $\begin{array}{l}\text { Pre-post: } T_{(9)}=-9.00 ; p<.001 \\
\text { Pre-seg: } T_{(9)}=-9.00 ; p<.001\end{array}$ & $\begin{array}{l}1.10 \\
1.10 \\
0.00\end{array}$ \\
\hline Frecuencia del habla & $\begin{array}{l}\text { Pretest } \\
\text { Postest } \\
\text { Seguimiento }\end{array}$ & $\begin{array}{c}1.00 \pm 2.11 \\
20.20 \pm 3.91 \\
20.20 \pm 3.91\end{array}$ & $\begin{array}{l}\text { Pre-post: } T_{(9)}=-13.52 ; p<.001 \\
\text { Pre-seg: } T_{(9)}=-13.52 ; p<.001\end{array}$ & $\begin{array}{l}8.32 \\
8.32 \\
0.00\end{array}$ \\
\hline \multicolumn{5}{|c|}{ Interacción Niño y Extraño } \\
\hline Habla no audible & $\begin{array}{l}\text { Postest } \\
\text { Postest } \\
\text { Seguimiento }\end{array}$ & $\begin{array}{l}0.00 \pm 0.00 \\
0.00 \pm 0.00 \\
0.00 \pm 0.00\end{array}$ & $\begin{array}{l}\text { (1) } \\
\text { (1) } \\
\text { (1) }\end{array}$ & $\begin{array}{l}0.00 \\
0.00 \\
0.00\end{array}$ \\
\hline Habla audible & $\begin{array}{l}\text { Pretest } \\
\text { Postest } \\
\text { Seguimiento }\end{array}$ & $\begin{array}{l}0.00 \pm 0.00 \\
1.60 \pm 0.52 \\
3.70 \pm 0.48\end{array}$ & $\begin{array}{c}\text { Pre-post: } T_{(9)}=-9.79 ; p<.001 \\
\text { Pre-seg: } T_{(9)}=-24.22 ; p<.001 \\
\text { Post-seg: } T_{(9)}=-9.00 ; p<.001\end{array}$ & $\begin{array}{l}1.46 \\
3.38 \\
3.69\end{array}$ \\
\hline $\begin{array}{l}\text { Habla audible } \\
\text { espontánea }\end{array}$ & $\begin{array}{c}\text { Pretest } \\
\text { Postest } \\
\text { Seguimiento }\end{array}$ & $\begin{array}{l}0.00 \pm 0.00 \\
0.70 \pm 0.48 \\
1.50 \pm 0.53\end{array}$ & $\begin{aligned} \text { Pre-post: } T_{(9)} & =-4.58 ; p=.001 \\
\text { Pre-seg: } T_{(9)} & =-9.00 ; p<.001 \\
\text { Post-seg: } T_{(9)} & =-6.00 ; p<.001\end{aligned}$ & $\begin{array}{l}0.64 \\
1.37 \\
1.52\end{array}$ \\
\hline Frecuencia del habla & $\begin{array}{l}\text { Pretest } \\
\text { Postest } \\
\text { Seguimiento }\end{array}$ & $\begin{array}{c}0.00 \pm 0.00 \\
7.00 \pm 3.20 \\
17.50 \pm 3.37\end{array}$ & $\begin{array}{l}\text { Pre-post: } T_{(9)}=-6.92 ; p<.001 \\
\text { Pre-seg: } T_{(9)}=-16.40 ; p<.001 \\
\text { Post-seg: } T_{(9)}^{(9)}=-11.39 ; p<.001\end{array}$ & $\begin{array}{c}1.40 \\
15.96 \\
3.28\end{array}$ \\
\hline \multicolumn{5}{|c|}{ Interacción Niño y Padres tras presencia del Extraño } \\
\hline Habla no audible & $\begin{array}{c}\text { Postest } \\
\text { Postest } \\
\text { Seguimiento }\end{array}$ & $\begin{array}{l}0.70 \pm 0.67 \\
0.50 \pm 0.53 \\
0.00 \pm 0.00\end{array}$ & $\begin{array}{l}\text { Pre-post: } T_{(9)}=1.00 ; \mathrm{p}=.343 \\
\text { Pre-seg: } T_{(9)}^{(9)}=3.28 ; p=.010 \\
\text { Post-seg: } T_{(9)}=3.00 ; p=.015\end{array}$ & $\begin{array}{l}0.27 \\
0.86 \\
0.95\end{array}$ \\
\hline Habla audible & $\begin{array}{l}\text { Pretest } \\
\text { Postest } \\
\text { Seguimiento }\end{array}$ & $\begin{array}{l}0.00 \pm 0.00 \\
4.50 \pm 0.71 \\
4.80 \pm 0.42\end{array}$ & $\begin{array}{l}\text { Pre-post: } T_{(9)}=-20.12 ; p<.001 \\
\text { Pre-seg: } T_{(9)}=-36.00 ; p<.001 \\
\text { Post-seg: } T_{(9)}^{(9)}=-1.96 ; p=.081\end{array}$ & $\begin{array}{l}4.11 \\
4.39 \\
0.37\end{array}$ \\
\hline $\begin{array}{l}\text { Habla audible } \\
\text { espontánea }\end{array}$ & $\begin{array}{c}\text { Pretest } \\
\text { Postest } \\
\text { Seguimiento }\end{array}$ & $\begin{array}{l}0.00 \pm 0.00 \\
2.60 \pm 1.07 \\
3.20 \pm 0.63\end{array}$ & $\begin{array}{l}\text { Pre-post: } T_{(9)}=-7.64 ; p<.001 \\
\text { Pre-seg: } T_{(9)}=-16.00 ; p<.001 \\
\text { Post-seg: } T_{(9)}^{(9)}=-2.71 ; p=.024\end{array}$ & $\begin{array}{l}2.38 \\
2.92 \\
0.51\end{array}$ \\
\hline Frecuencia habla & $\begin{array}{l}\text { Pretest } \\
\text { Postest } \\
\text { Seguimiento }\end{array}$ & $\begin{array}{c}2.10 \pm 1.91 \\
24.60 \pm 5.52 \\
28.80 \pm 3.99\end{array}$ & $\begin{array}{l}\text { Pre-post: } T_{(9)}=-14.37 ; p<.001 \\
\text { Pre-seg: } T_{(9)}=-26.98 ; p<.001 \\
\text { Post-seg: } T_{(9)}=-4.47 ; p=.002\end{array}$ & $\begin{array}{c}10.77 \\
12.78 \\
0.70\end{array}$ \\
\hline
\end{tabular}

(1) No se puede calcular porque el error típico de la diferencia es $0 \mathrm{EHEx}=$ Test situacional Diferencias en la comorbilidad El único trastorno comórbido que alcanzó diferencias significativas fue el Trastorno de ansiedad por separación $\left(Q_{(2)}=7.60 ; p=.02\right)$. 


\section{Satisfacción con el tratamiento}

La satisfacción con la aplicación de la intervención de los padres $(M=27.20 ; D T=1.23)$ y de los profesores $(M=28.00 ; D T=0.94)$ fue alta (rango: $8-32)$.

\section{Discusión}

El conjunto de los datos proporcionados por las medidas de las variables dependientes, tanto provenientes de la entrevista clínica (incluido el índice de severidad), como los de los instrumentos de autoinforme (incluida la satisfacción de padres y profesores) y el test situacional, son coherentes entre sí y muestran la bondad de los efectos de la IPMSEC, en su aplicación en el contexto donde se presenta el MS (el centro educativo), cuando el niño todavía habla en algún momento y de algún modo a su profesor.

Además, estos resultados son coherentes con las conclusiones de las revisiones realizadas por Cohan, Chavira \& Stein (2006), Muris \& Ollendick (2015), Viana, Beidel \& Rabian (2009) o Zakszeski \& DuPaul (2017), así como con las de Lang et al. (2016) respecto del mantenimiento de los efectos del tratamiento en las medidas de seguimiento de la efectividad del tratamiento cognitivo-conductual. En nuestro caso, éstas siguen mostrando un año después el incremento de las mejoras en todas las medidas.

Es cierto que, como recordaba Lang et al. (2016), a diferencia de los estudios controlados aleatorizados (Bergman et al., 2013; Oerbeck et al., 2012, 2015, 2018), cuyo número de sesiones de tratamiento y la duración de éste viene determinado a priori por el manual, en intervenciones derivadas de protocolos inespecíficos como la suya (Chorpita, 2007; Lang et al., 2016) o específicos para el MS como el presente, lo que se persigue es eliminar el trastorno, no probar que es mejor tratar que no hacerlo (eficacia). Es decir, probar en qué medida el tratamiento es efectivo independientemente de su duración y de los componentes utilizados en su aplicación (eficiencia).

No obstante, como podemos observar, los datos muestran que la duración media de los 10 casos tratados en esta investigación tampoco es desproporcionada, dado que ésta fue de 15.18 semanas (cinco días lectivos/semana) y la desviación típica de 3.67. Además, estos datos están en línea con las conclusiones de Khan, Parsonage \& Stubbs (2015) cuando, en su informe sobre la eficiencia de la intervención psicológica temprana en los centros educativos, señalan que entre las intervenciones eficaces para el tratamiento de los trastornos de conducta en niños y adolescentes ésta destaca por la relación costes/beneficios con una proporción de 27:1.

Desde nuestro punto de vista, los tamaños del efecto mostrados por la IPMSCE, tras su aplicación y en la medida de seguimiento, pueden explicarse por el hecho de que el tratamiento se adecúa a las variables que resultan más relevantes en el mantenimiento del MS, entre las que se incluyen las estructurales (disposicionales) propias del ámbito institucional y las estrictamente individuales. Es decir, para que un tratamiento resulte efectivo cuando se aplica allí donde el trastorno se presenta ha de abarcar el ámbito institucional (infraestructura humana y material — familias y escuela-) y el individual (variables relevantes en el mantenimiento del MS).

Hemos de destacar el papel que atribuimos a las estrategias que incluye la IPMSCE (transmisión de información, la reestructuración cognitiva, las instrucciones y el modelado) como componentes necesarios del tratamiento de los adultos que interactúan cotidianamente con el niño con MS. Con su aplicación se pretende modificar las creencias y la desinformación que afecta al modo de relacionarse con los mudos selectivos (véase Olivares-Olivares \& Olivares, 2018), así como capacitarlos mediante el modelado para que sepan manejar adecuadamente las variables implicadas en las situaciones en las que el MS se presenta (las propias y las del niño).

De igual modo, hipotetizamos que ha jugado un papel muy relevante en los resultados obtenidos la planificación del enfrentamiento gradual de los niños al conjunto de las situaciones sociales temidas por ellos (relativas al empleo del habla en el centro educativo), diseñando y aplicando el entrenamiento centrado en el desvanecimiento estimular (para facilitar todo lo posible la generalización del habla), la economía de fichas (para modificar el aislamiento social y la implicación de los compañeros), el coste de respuesta (para intensificar la "presión de grupo") y su exposición a la reproducción pública de una grabación audiovisual (modificar expectativas de resultado de los compañeros, debilitar la estrategia de evitación del mudo selectivo e incrementar la probabilidad de que surjan oportunidades de relación social verbal). 
También hemos de subrayar el papel que, desde nuestro punto de vista, ha desempeñado el empleo de los resultados del sociograma para confeccionar una prelación, en base al número de elecciones, que ayude a reducir la magnitud de la intensidad del efecto de las incorporaciones de compañeros en la inhibición/bloqueo del habla audible.

Otro tanto postulamos respecto del papel del juego cooperativo en los resultados obtenidos, dado su papel mediador y vertebrador de la aplicación del tratamiento.

Por último, entre las limitaciones del presente trabajo se hallan las propias del diseño pre-experimental utilizado y las relativas a la validez externa. Así, pese a que hemos procurado maximizar las estrategias de control para que los efectos del tratamiento se deban principalmente a éste y no a otras variables extrañas, siguiendo las sugerencias de la Task Force on Promotion and Dissemination of Psychological Procedures (1995), no es posible afirmar una relación exclusiva y unívoca entre el tratamiento aplicado y los cambios medidos, más allá de lo que permiten las pruebas utilizadas. Respecto de la validez externa hemos de indicar que nuestros hallazgos solo son generalizables a niños con MS que hablan al menos en alguna ocasión a su profesor y presentan una demora inferior a los dos años entre el inicio del MS y el de su tratamiento.

\section{Referencias}

American Psychiatric Association (1994). Diagnostic and Statistical Manual of Mental Disorders (DSM-IV). Washington, D.C.: APA. (traducido al español en 1995).

American Psychiatric Association (2013). Diagnostic and statistical manual of mental disorders (5 ${ }^{\mathrm{a}}$ ed.). Arlington, VA: Autor.

Attkisson, C. C., \& Zwick, R. (1982). The client satisfaction questionnaire: psychometric properties and correlations with service utilization and psychotherapy outcome. Evaluation and Program Planning, 5, 233-237.

Ato, M., López, J. J., \& Benavente, A. (2013). Un sistema de clasificación de los diseños de investigación en psicología. Anales de psicología, 29, 1038-1059.

Balbuena-Teruel, S., Rueda-Lozano, I., \& López-Herrero, P. (2012). Estudio de un caso de mutismo selectivo: evaluación e intervención en el contexto escolar. Análisis y Modificación de Conducta, 38, 71-88.

Barrasa, A., \& Gil, F. (2004). Un programa informático para el cálculo y la representación de índices y valores sociométricos. Psicothema, 16, 329-335.

Bergman, R. L., Gonzalez, A., Piacentini, J., \& Keller, M. L. (2013). Integrated behavior therapy for selective mutism: A randomized controlled pilot study. Behaviour Research and Therapy, 51, 680-689.

Bergman, R. L., Keller, M. L., Piacentini, J., \& Bergman, A. J. (2008). The development and psychometric properties of the Selective Mutism Questionnaire. Journal of Clinical Child and Adolescent Psychology, 37, 456-464.

Bergman, R. L., Keller, M., Wood, J., Piacentini, J. y McCracken, J. (2001). Selective Mutism Questionnaire: Development and findings. Proceedings of the American Academy of Child and Adolescent Psychiatry Meeting, 48, 163.

Bulotsky-Shearer, R. J., Fantuzzo, J. W., \& McDermott, P. A. (2008). An investigation of classroom situational dimensions of emotional and behavioral adjustment and cognitive and social outcomes for Head Start children. Developmental Psychology, 44, 139-154.

Busse, R. T., \& Downey, J. (2011). Selective mutism: A three-tiered approach to prevention and intervention. Contemporary School Psychology, 15, 53-63.

Cautela, J. R., \& Brion-Meisels, L. (1979). A children's reinforcement survey schedule. Psychological Reports, 44, $327-338$.

Chorpita, B. F. (2007). Modular cognitive-behavioral therapy for childhood anxiety disorders. New York: Guilford.

Cohan, S. L., Chavira, D. A., \& Stein, M. B. (2006). Practitioner review: Psychosocial interventions for children with selective mutism: A critical evaluation of the literature from 1990-2005. Journal of Child Psychology and Psychiatry, 47, 1085-1097.

Cohen, J. (1988). Statistical power analysis for the behavioral sciences (2nd ed.). Hillsdale, Nueva Jersey: Erlbaum.

García-Bacete, F.J., \& González, J. (2010). SOCIOMET. Evaluación de la competencia social entre iguales: La sociometría y otras medidas. Madrid: TEA.

González, J., \& García-Bacete, F. J. (2010). SOCIOMET. Manual de uso: Programa para la realización de estudios sociométricos. Madrid: TEA.

Hargreaves, W. A. y Attkisson, C. C. (1978). Evaluating program outcomes. En C. C. Attkisson, W. A. Hargreaves, M. J. Horowitz y J. E. Sorensen (eds.), Evaluation of human service programs (pp. 303-339). New York: Academic Press. 
IBM Corporation (2017). SPSS 25. Chicago, Il: Autor.

Khan, L., Parsonage, M., \& Stubbs, J. (2015). Investing in children's mental health. A review of evidence on the costs and benefits of increased service provision. Recuperado desde https://www.centreformentalhealth.org.uk/pdfs/investing_in_childrens_mental_health.pdf

Lang, C., Ziv, N., Gothelf, A., Domachevsky, S., Ginton, L., Kuhsnir, J., \& Gothelf, D. (2016). The outcome of children with selective mutism following cognitive behavioral intervention: A follow-up. European Journal of Pediatrics, 75, 481-487.

Letamendi, A. M., Chavira, D. A., Hitchcock, C. A., Roesch, S. C., Shipon-Blum, E. y Stein, M. B. (2008). Selective mutism questionnaire: measurement structure and validity. Journal of the American Academy of Child \& Adolescent Psychiatry, 47(10), 1197-1204.

Muris, P., \& Ollendick, T. H. (2015). Children who are anxious in silence: A review on selective mutism, the new anxiety disorder in the DSM-5. Clinical Child and Family Psychology Review, 18, 151-169.

Oerbeck, B., Johansen, J., Lundahl, K., \& Kristensen, H. (2012). Selective mutism: A home-and kindergarten-based intervention for children 3-5 years: A pilot study. Clinical Child Psychology and Psychiatry, 17, 370-383.

Oerbeck, B., Overgaard, K. R., Stein, M. B., Pripp, H., \& Kristensen, H. (2018). Treatment of selective mutism: a 5year followup study. European Child and Adolescent Psychiatry, 27, 997-1009.

Oerbeck, B., Stein, M. B., Pripp, A. H., \& Kristensen, H. (2015). Selective mutism: Follow-up study 1 year after end of treatment. European Child and Adolescent Psychiatry, 24, 757-766.

Olivares, J. (1994). El niño con miedo a hablar. Madrid: Pirámide

Olivares, J. y Olivares-Olivares, P. J. (2008a). Evaluación de las expectativas de resultado de los compañeros de clase. Unidad de terapia de conducta. Facultad de Psicología. Universidad de Murcia.

Olivares, J., \& Olivares-Olivares, P. J. (2008b). Manual para la intervención psicológica en niños con mutismo selectivo en los centros educativos (IPMSCE). Manuscrito no publicado. Departamento de Personalidad, Evaluación y Tratamiento Psicológicos. Universidad de Murcia.

Olivares, J., \& Olivares-Olivares, P. J. (2008c). Prueba para evaluar el habla en presencia de/con extraños en niños con mutismo selectivo (EHEx). Manuscrito no publicado. Departamento de personalidad, evaluación y tratamiento psicológicos. Facultad de psicología. Universidad de Murcia.

Olivares, J., \& Olivares-Olivares, P. J. (2018). Mutismo selectivo. Naturaleza, evaluación y tratamiento. Madrid: Pirámide

Olivares-Olivares, P. J., \& Olivares, J. (2018). Actualización de un modelo tentativo del mutismo selectivo. Behavioral Psychology/ Psicologia Conductual, 26, 115-140.

Olivares-Olivares, P. J., Macià, D., \& Olivares, J. (2019). Tratamiento del mutismo selectivo en niñas políglotas. Behavioral Psychology/ Psicologia Conductual, 27, 123-147.

Pérez, A. R., \& Bello, M. D. M. (2001). El sociograma: estudio de las relaciones informales en las organizaciones. Madrid: Pirámide. Silverman, W. K., Albano, A. M., \& Sandín, B. (2003). Entrevista para el diagnóstico de los trastornos de ansiedad en niños (ADIS-IV: P): Entrevista para los padres. Madrid: Klinik.

Silverman, W. K., Saavedra, L. M. y Pina, A. A. (2001). Test-retest reliability of anxiety symptoms and diagnoses with the anxiety disorders interview schedule for DSM-IV: child and parent Versions. Journal of the American Academy of Child \& Adolescent Psychiatry, 40, 937-944.

Task Force on Promotion and Dissemination of Psychological Procedures (1995). Training and dissemination of empirically-validated psychosocial treatments: Report and recommendations. The Clinical Psychologist, 48, 3-23.

Viana, A. G., Beidel, D. C., \& Rabian, B. (2009). Selective mutism: A review and integration of the last 15 years. Clinical Psychology Review, 29, 57-67.

Zakszeski, B. N., \& DuPaul, G. J. (2017). Reinforce, shape, expose, and fade: a review of treatments for selective mutism (20052015). School Mental Health, 9, 1-15. 